\begin{tabular}{c|c|c} 
y & $\begin{array}{c}\text { Journal of the Egyptian Mathematical Society } \\
\text { Volume (26) - Issue (2) - } 2018\end{array}$ & ISSN:1110-256X \\
\hline
\end{tabular}

Algorithmic

\title{
STATISTICAL INFERENCE AND PREDICTION FOR THE GOMPERTZ DISTRIBUTION BASED ON MULTIPLY TYPE-I CENSORED DATA
}

\author{
M. M. Mohie El-Din ${ }^{1}$ and M. H. Abu-Moussa ${ }^{2}$ \\ ${ }^{1}$ Department of Mathematics, Faculty of Science, Al-Azhar University, Cairo-Egypt \\ ${ }^{2}$ Department of Mathematics, Faculty of Science, Cairo University, Giza-Egypt \\ Received 4/1/2018Ｒevised 28/1/2018Ａccepted 3/5/2018
}

\begin{abstract}
In this paper, we make a statistical inference for the Gompertz distribution based on multiply Type-I censored data, where we determine a censoring time point for each unit in the life time test. Estimation of the parameters is obtained using maximum likelihood method and Bayesian method, also the asymptotic confidence intervals for the parameters are constructed based on the approximate of the Fisher information matrix. The necessary condition for existence and uniqueness of the maximum likelihood estimators is discussed. The Bayesian estimates are obtained depending on the squared error loss function, linear exponential loss function and the generalized entropy loss function. The One-sample Bayesian prediction intervals are constructed for the unobserved lifetimes in the same sample. A real data example is presented to illustrate the methods of inference developed here. Finally, the simulation study is executed to compare the performance of the proposed methods.
\end{abstract}

Keywords: Statistical Inference; Multiply Type-I Censored Data; Gompertz distribution; Maximum likelihood estimator; Bayesian estimation; One-Sample Bayesian prediction intervals

AMS 2000 Subject Classification: 62N02, 62N01, 62N05, 62F15.

\section{Introduction}

Due to cost and time limitations, most of the experiments that tend to measure the life time and reliability of items or devices must be terminated before all units have failed. For these reasons, statisticians depend on censored sample in their work. Type-I and Type-II censoring are the most two popular censoring schemes. In Type-I the experiment is terminated based on only one pre-specified time point and the survival units are removed from the test at this point, while in Type-II the experiment is stopped at the time of a particular observation, and the survival units are removed at this point. Another type of censoring is the progressive Type-I censoring, where the experimenter determines several time points and several units are terminated at each time point until the largest pre-specified time point has been reached. Also at each time point the experimenter can remove a particular number of survival units. Readers can see Balakrishnan and Aggarwala [1] and Balakrishnan [2] who presented a study on different features of progressive censoring schemes. The generalization of Type-I censoring is that all the units in life test are terminated at different times and it is defined as multiple Type-I censoring.

*mhmoussa@sci.cu.edu.eg, corresponding author 
In this type, every unit is restricted by its pre-specified time point only. If the lifetime of the unit is less than or equal to its time point then it fails, and it will survive if its lifetime exceeds the time point. Jia et al. [3] makes an inference on the reliability of the Weibull distribution with multiply Type-I censored data. Also they have studied the exact inference for exponential distribution with multiply Type-I censored data [4]).

In life testing, we may have unobserved failure times for some units, so prediction intervals are used to predict by these failures. In prediction intervals, we depend on the previous data to predict the future observations with a specified probability. Prediction intervals were discussed by many authors, examples of these papers are as follows: Mohie El-Din and Shafay [5], obtained the Bayesian prediction intervals depending on progressively Type-II censored data. Also Bayesian prediction intervals based on the Type-I hybrid censored data were studied by Shafay and Balakrishnan [6]. Mohie El-Din et al. [7] studied the Bayesian prediction intervals of generalized order statistics based on multiply Type-II censored data. Also Mohie El-Din et al. [8] used the record data to make statistical inference and prediction for the inverse Weibull distribution. See also [9] and [10].

The Gompertz distribution is one of the most important distributions for modeling lifetimes and reliability. It was introduced in 1825 by Benjamin Gompertz [11]to describe human mortality and establish actuarial tables. The probability density function (PDF) of the Gompertz distribution, denoted by $\operatorname{GOM}(\alpha, \beta)$, is

$$
f(x ; \alpha, \beta)=\beta \exp \left[\alpha x-\frac{\beta}{\alpha}\left(e^{\alpha x}-1\right)\right], \quad x \geq 0, \alpha, \beta>0,
$$

where $\mathrm{x}$ is the failure time and $\alpha$ and $\beta$ are the scale and shape parameters respectively. The cumulative distribution function $(\mathrm{CDF})$ is

$$
F(x ; \alpha, \beta)=1-\exp \left[-\frac{\beta}{\alpha}\left(e^{\alpha x}-1\right)\right] .
$$

also the reliability function $R(x)$ and the failure rate function $h(x)$ are given by,

$$
\begin{aligned}
R(x) & =\exp \left[-\frac{\beta}{\alpha}\left(e^{\alpha x}-1\right)\right] \\
h(x) & =\beta e^{\alpha x}
\end{aligned}
$$

The Gompertz distribution can be transformed to exponential distribution with mean $1 / \beta$, when we use the transformation $Y=\frac{1}{\alpha}\left(e^{\alpha x}-1\right)$. Many authors discussed this distribution in their work as Pollard et al. [12], they study the Gompertz distribution and its applications, Chen [13] obtained the parameter estimation of the Gompertz distribution. Chang and Tsai [14] introduced point and interval estimation for the Gompertz distribution under progressive Type-II while, Wu et al. [15] gives the parameter estimates for the Gompertz distribution using the least squares method. Recently Mohie El-Din et al. [16] made statistical inference for the Gompertz distribution based on progressive type-II hybrid censored data.

The aim from this work is to make statistical inference for $\operatorname{GOM}(\alpha, \beta)$ based on multiply Type-I censoring. In this article, we obtain the maximum likelihood estimates (MLEs) of the parameters $\alpha$ and $\beta$. Also, we construct the asymptotic confidence intervals of the parameters. The Bayesian estimation (BE) have been introduced using gamma priors for the unknown parameters under the squared error loss function $(S E L)$, the linear exponential loss function (LINEX) and the generalized entropy (GE) loss function. Also we construct the one-sample Bayesian prediction intervals to predict a future observation from the same sample. We also discuss the proposed methods using a real data example. Finally, we compare the estimators by applying the Monte Carlo simulation. 
The rest of the paper is organized as follows. In section 2, the likelihood function $L(x)$ is formulated, then the MLEs of the parameters are obtained, also the existence and uniqueness of the MLEs is discussed and the asymptotic confidence intervals are obtained. BE are studied in section 3. Also the MCMC method is applied in section 4. The one-sample Bayesian prediction intervals are derived in section 5. A real data example is constructed in section 6 , while the simulation study is conducted in section 7 . Finally the paper is concluded in section 8 .

\section{Likelihood function and MLE}

Suppose that we have a lifetime test with $n$ units whose lifetimes $t_{1}, t_{2}, \ldots, t_{n}$ follow $\operatorname{GOM}(\alpha, \beta)$. Based on multiply Type-I censoring, we determine a time point for each unit in the test, so we have $\mathrm{n}$ censoring time points $T_{1}, T_{2}, \ldots, T_{n}$, so the sample observations are $x_{1}, x_{2}, \ldots, x_{n}$, where $x_{i}=t_{i} \delta_{i}+T_{i}\left(1-\delta_{i}\right)$ and

$$
\delta_{i}=\left\{\begin{array}{cc}
1, & t_{i} \leq T_{i} ; \\
0, & t_{i}>T_{i} .
\end{array}, i=1,2, \ldots, n .\right.
$$

which means that the observation $x_{i}$ is the failure time if $\delta_{i}=1$, if $\delta_{i}=0$ then the failure time $t_{i}$ for this unit exceed the time point $T_{i}$ and the observation $x_{i}$ is the censored time $T_{i}$.

For example, if $n=5$ and $\delta_{2}=1$ then $x_{2}=t_{2}$ which means that the second unit fails in time interval $\left(0, T_{2}\right]$. If $\delta_{4}=0$ then $x_{4}=T_{4}$ (censored time), which means that the fourth unit survives in time interval $\left(0, T_{4}\right]$.

The likelihood function is given by

$$
L(x ; \alpha, \beta)=\prod_{i=1}^{n}\left[f\left(x_{i}\right)\right]^{\delta_{i}}\left[1-F\left(x_{i}\right)\right]^{1-\delta_{i}} .
$$

Using (1) and (2), then the likelihood function becomes

$$
L(x ; \alpha, \beta)=\beta^{r} \exp \left[\alpha \sum_{i=1}^{n} x_{i} \delta_{i}-\frac{\beta}{\alpha} \sum_{i=1}^{n}\left(e^{\alpha x_{i}-1}\right)\right]
$$

where $r$ is the number of observations(failures) and equal $\sum_{i=1}^{n} \delta_{i}$. Taking the logarithm for (4), then the log-likelihood function is

$$
l(x ; \alpha, \beta)=r \times \log (\beta)+\alpha \sum_{i=1}^{n} x_{i} \delta_{i}-\frac{\beta}{\alpha} \sum_{i=1}^{n}\left(e^{\alpha x_{i}-1}\right) .
$$

By applying the partial derivatives for (5) with respect to $\alpha$ and $\beta$ and putting the derivatives equal to zero, then we get:

$$
\hat{\beta}=\frac{r \alpha}{\sum_{i=1}^{n}\left(e^{\alpha x_{i}}-1\right)},
$$

and

$$
\sum_{i=1}^{n} x_{i} \delta_{i}+\frac{\beta}{\alpha^{2}} \sum_{i=1}^{n}\left(e^{\alpha x_{i}}-1\right)-\frac{\beta}{\alpha} \sum_{i=1}^{n} x_{i} e^{\alpha x_{i}}=0,
$$

Substituting by (6) in (7), then we get

$$
J(\alpha)=\sum_{i=1}^{n} x_{i} \delta_{i}+r\left(\frac{1}{\alpha}-\frac{\sum_{i=1}^{n} x_{i} e^{\alpha x_{i}}}{\sum_{i=1}^{n}\left(e^{\alpha x_{i}}-1\right)}\right)=0 .
$$

The MLE of $\alpha, \hat{\alpha}_{M L}$ is obtained by solving equation (8), we get the MLE of $\beta, \hat{\beta}_{M L}$ by using $\hat{\alpha}_{M L}$ in (6). Substituting by $\hat{\alpha}_{M L}$ and $\hat{\beta}_{M L}$ in (3), then we get the MLE of the reliability function $\hat{R}(x)$ and hazard function $\hat{h}(x)$. 


\subsection{The Existence and uniqueness of the MLEs}

Now, we will discuss the existence and uniqueness of the MLE estimates. From equation (6), we can see that the MLE of $\beta$ has an explicit form that depend on the MLE of the parameter $\alpha$, which is obtained by solving equation (8). The function $J(\alpha)$ is a non-linear function, so we can not obtain an explicit formula for the MLE of $\alpha$. The following theorem will give the condition that ensures the existence and uniqueness of the MLE estimates of the parameter $\alpha$ and $\beta$.

Theorem 1. Let $x_{1} \leq x_{2} \leq \ldots \leq x_{r}<x_{r+1} \leq \ldots \leq x_{n}$ be the observed sample based on multiply Type-I censoring and follow $\operatorname{GOM}(\alpha, \beta)$. Then using equations (6) and (8), the MLEs $\hat{\alpha}$ and $\hat{\beta}$ of the parameters $\alpha$ and $\beta$ exist and are unique, if and only if

$$
2\left(\sum_{i=1}^{n} x_{i}\right)\left(\sum_{i=1}^{n} x_{i} \delta_{i}\right)>r \sum_{i=1}^{n} x_{i}^{2} .
$$

proof Mohie El-Din et al. [16] and Ghitany et al. [17] studied the existence and uniqueness of the MLEs for the parameters of $\operatorname{GOM}(\alpha, \beta)$ based on progressive Type-II censored samples. They proved that $J(\alpha)$ is a decreasing function by showing that its derivative is a negative function, then they proved that $J(\alpha=0)>0$ and $J(\alpha=\infty)<0$, which mean that $J(\alpha)$ has a unique root in $(0, \infty)$, therefore $\hat{\beta}$ also is exist and unique. Also, they proved that $\hat{\alpha}$ and $\hat{\beta}$ indeed maximizes the function $l$ in (5). The proof in our case is simple when we follow the steps in the proof of Ghitany et al. [17].

\subsection{Asymptotic confidence intervals}

in this subsection, we derive the asymptotic confidence intervals for the parameters $\alpha$ and $\beta$. We begin with the observed fisher information matrix, see [18], which is given as follows,

$$
\hat{I}(\alpha, \beta)=-\left[\begin{array}{cc}
\frac{\partial^{2} l}{\partial \alpha^{2}} & \frac{\partial^{2} l}{\partial \alpha \partial \beta} \\
\frac{\partial^{2} l}{\partial \beta \partial \alpha} & \frac{\partial^{2} l}{\partial \beta^{2}}
\end{array}\right]
$$

where

$$
\begin{aligned}
\frac{\partial^{2} l}{\partial \alpha^{2}} & =-\frac{2 \beta \sum_{i=1}^{n}\left(e^{\alpha x_{i}}-1\right)}{\alpha^{3}}+\frac{2 \beta \sum_{i=1}^{n} x_{i} e^{\alpha x_{i}}}{\alpha^{2}}-\frac{\beta \sum_{i=1}^{n} x_{i}^{2} e^{\alpha x_{i}}}{\alpha} \\
\frac{\partial^{2} l}{\partial \beta^{2}} & =\frac{-r}{\beta^{2}} \\
\frac{\partial^{2} l}{\partial \beta \partial \alpha} & =\frac{\partial^{2} l}{\partial \alpha \partial \beta}=\frac{\sum_{i=1}^{n}\left(e^{\alpha x_{i}}-1\right)}{\alpha^{2}}-\frac{\sum_{i=1}^{n} x_{i} e^{\alpha x_{i}}}{\alpha}
\end{aligned}
$$

Then the observed asymptotic Variance-Covariance matrix is the inverse matrix of (10) which is given by,

$$
\hat{V}=[\hat{I}(\hat{\alpha}, \hat{\beta})]^{-1}=\left[\begin{array}{cc}
\hat{\operatorname{Var}}(\hat{\alpha}) & \hat{\operatorname{Cov}}(\hat{\alpha}, \hat{\beta}) \\
\hat{\operatorname{Cov}}(\hat{\alpha}, \hat{\beta}) & \hat{\operatorname{Var}}(\hat{\beta})
\end{array}\right]
$$

Then the asymptotic confidence interval for the parameters $\alpha$ and $\beta$ is given by,

$$
\hat{\alpha} \pm Z_{\eta / 2} \sqrt{\hat{\operatorname{Var}(\hat{\alpha})}} \quad \text { and } \quad \hat{\beta} \pm Z_{\eta / 2} \sqrt{\hat{\operatorname{Var}(\hat{\beta})}}
$$

with $100(1-\eta) \%$ confidence degree; where $Z_{\eta / 2}$ is obtained from the table of the standard normal distribution. 


\section{Bayesian Estimation (BE)}

In this section, we will obtain the $\mathrm{BE}$ for the parameters of the $G O M(\alpha, \theta)$ based on multiply Type-I censoring using the SEL loss function as a symmetric loss function which is defined as, assume $u(\alpha, \beta)$ is a function in $\alpha$ and $\beta$

$$
L_{1}(u(\alpha, \beta), \widehat{u}(\alpha, \beta))=(\widehat{u}(\alpha, \beta)-u(\alpha, \beta))^{2},
$$

where $\widehat{u}(\alpha, \beta)$ is an estimate of $u(\alpha, \beta)$. The BE for the loss function $L_{1}$ is the posterior mean $\widehat{u}(\alpha, \beta)_{B S}=E[u(\alpha, \beta) \mid \underline{x}]$, while we use the LINEX loss function as an asymmetric loss function and defines as

$$
L_{2}(u(\alpha, \beta), \widehat{u}(\alpha, \beta))=e^{h(\widehat{u}(\alpha, \beta)-u(\alpha, \beta))}-h(\widehat{u}(\alpha, \beta)-u(\alpha, \beta))-1, \quad h \neq 0,
$$

and the BE for any parameter $u(\alpha, \beta)$ under the loss function $L_{2}$ is given as,

$$
\widehat{u}(\alpha, \beta)_{B L}=-\frac{1}{h} \log \left[E\left(e^{-h u(\alpha, \beta)} \mid \underline{x}\right)\right]
$$

Another asymmetric loss function is GE, which is given by,

$$
L_{3}(u(\alpha, \beta), \widehat{u}(\alpha, \beta))=\left(\frac{\widehat{u}(\alpha, \beta)}{u(\alpha, \beta)}\right)^{q}-q \log \left(\frac{\widehat{u}(\alpha, \beta)}{u(\alpha, \beta)}\right)-1, q \neq 0,
$$

and its $\mathrm{BE}$ is given by,

$$
\widehat{u}(\alpha, \beta)_{B E}=\left(E\left[u(\alpha, \beta)^{-q} \mid \underline{x}\right]\right)^{-1 / q},
$$

Assume that the parameters $\alpha$ and $\beta$ are independent variables having gamma prior distributions $\pi_{1}(\alpha)$ and $\pi_{2}(\beta)$ respectively.

$$
\begin{aligned}
& \pi_{1}(\alpha)=\frac{b_{1}^{a_{1}}}{\Gamma\left[a_{1}\right]} \alpha^{a_{1}-1} e^{-b_{1} \alpha} \\
& \pi_{2}(\beta)=\frac{b_{2}^{a_{2}}}{\Gamma\left[a_{2}\right]} \beta^{a_{2}-1} e^{-b_{2} \beta}
\end{aligned}
$$

where $a_{1}, b_{1}, a_{2}, b_{2}>0$ are the hyper parameters. Using the likelihood function in (4)and the priors of $\alpha, \beta$ in (13), then the density of the posterior distribution is given by,

$$
\begin{aligned}
\pi^{*}(\alpha, \beta \mid \underline{X}) & =\frac{L(\alpha, \beta) \cdot \pi(\alpha) \pi(\beta)}{\int_{0}^{\infty} \int_{0}^{\infty} L(\alpha, \beta) \cdot \pi(\alpha) \pi(\beta) d \alpha d \beta} \\
& \propto \beta^{r+a_{2}-1} \alpha^{a_{1}-1} \exp \left[\alpha\left(\sum_{i=1}^{n} x_{i} \delta_{i}-b_{1}\right)-\beta\left(\frac{1}{\alpha} \sum_{i=1}^{n}\left(e^{\alpha x_{i}}-1\right)+b_{2}\right)\right]
\end{aligned}
$$

The BEs for the parameters $\alpha$ and $\beta$ under the SEL loss function $L_{1}$ are given by,

$$
\begin{aligned}
& \hat{\alpha}_{B S}=E[\alpha \mid \underline{x}]=\int_{0}^{\infty} \int_{0}^{\infty} \beta^{r+a_{2}-1} \alpha^{a_{1}} \exp \left[\alpha\left(\sum_{i=1}^{n} x_{i} \delta_{i}-b_{1}\right)-\beta\left(\frac{1}{\alpha} \sum_{i=1}^{n}\left(e^{\alpha x_{i}}-1\right)+b_{2}\right)\right] d \alpha d \beta . \\
& \hat{\beta}_{B S}=E[\beta \mid \underline{x}]=\int_{0}^{\infty} \int_{0}^{\infty} \beta^{r+a_{2}} \alpha^{a_{1}-1} \exp \left[\alpha\left(\sum_{i=1}^{n} x_{i} \delta_{i}-b_{1}\right)-\beta\left(\frac{1}{\alpha} \sum_{i=1}^{n}\left(e^{\alpha x_{i}}-1\right)+b_{2}\right)\right] d \alpha d \beta .
\end{aligned}
$$

For the LINEX loss function $L_{2}$, the BEs for $\alpha$ and $\beta$ are given by,

$$
\hat{\alpha}_{B L}=-\frac{1}{h} \log \left(E\left[e^{-h \alpha} \mid \underline{x}\right]\right), h \neq 0
$$


where

$$
\begin{gathered}
E\left[e^{-h \alpha} \mid \underline{x}\right]=\int_{0}^{\infty} \int_{0}^{\infty} \beta^{r+a_{2}-1} \alpha^{a_{1}-1} \exp \left[\alpha\left(\sum_{i=1}^{n} x_{i} \delta_{i}-b_{1}-h\right)-\beta\left(\frac{1}{\alpha} \sum_{i=1}^{n}\left(e^{\alpha x_{i}}-1\right)+b_{2}\right)\right] d \alpha d \beta . \\
\hat{\beta}_{B L}=-\frac{1}{h} \log \left(E\left[e^{-h \beta} \mid \underline{x}\right]\right), h \neq 0
\end{gathered}
$$

where

$$
E\left[e^{-h \beta} \mid \underline{x}\right]=\int_{0}^{\infty} \int_{0}^{\infty} \beta^{r+a_{2}-1} \alpha^{a_{1}-1} \exp \left[\alpha\left(\sum_{i=1}^{n} x_{i} \delta_{i}-b_{1}\right)-\beta\left(\frac{1}{\alpha} \sum_{i=1}^{n}\left(e^{\alpha x_{i}}-1\right)+b_{2}+h\right)\right] d \alpha d \beta .
$$

The BEs for $\alpha$ and $\beta$ depending on the GE loss function $L_{3}$ is given by,

$$
\hat{\alpha}_{B E}=E\left(\alpha^{-q} \mid \underline{x}\right)^{-1 / q},
$$

where

$$
\begin{gathered}
E\left[\alpha^{-q} \mid \underline{x}\right]=\int_{0}^{\infty} \int_{0}^{\infty} \beta^{r+a_{2}-1} \alpha^{a_{1}-q-1} \exp \left[\alpha\left(\sum_{i=1}^{n} x_{i} \delta_{i}-b_{1}\right)-\beta\left(\frac{1}{\alpha} \sum_{i=1}^{n}\left(e^{\alpha x_{i}}-1\right)+b_{2}\right)\right] d \alpha d \beta . \\
\hat{\beta}_{B E}=E\left(\beta^{-q} \mid \underline{x}\right)^{-1 / q},
\end{gathered}
$$

where

$$
E\left(\beta^{-q} \mid \underline{x}\right)==\int_{0}^{\infty} \int_{0}^{\infty} \beta^{r+a_{2}-q-1} \alpha^{a_{1}-1} \exp \left[\alpha\left(\sum_{i=1}^{n} x_{i} \delta_{i}-b_{1}\right)-\beta\left(\frac{1}{\alpha} \sum_{i=1}^{n}\left(e^{\alpha x_{i}}-1\right)+b_{2}\right)\right] d \alpha d \beta .
$$

Unfortunately, These integrals cannot be computed explicitly. Therefore, the approximated values for these integrals are computed using the MCMC method.

\section{MCMC Algorithm for Bayesian Estimation}

In this section, we consider the MCMC algorithm to get an approximate for BEs for the parameters $\alpha$ and $\beta$ under multiply Type-I censoring. We consider the Metropolis-Hastings algorithm to generate samples from the conditional posterior distributions, then we compute the BEs. For more details about the MCMC methods, the reader can see, Robert and Casella [19], Upadhyaya and Gupta [20], and Jaheen and Al Harbi [21]. Using (14), the conditional posterior distributions of $\alpha$ and $\beta$, can be obtained as follows,

$$
\pi_{1}^{*}(\alpha \mid \beta, \underline{X}) \propto \alpha^{a_{1}-1} \exp \left[\alpha\left(\sum_{i=1}^{n} x_{i} \delta_{i}-b_{1}\right)-\beta\left(\frac{1}{\alpha} \sum_{i=1}^{n}\left(e^{\alpha x_{i}}-1\right)+b_{2}\right)\right]
$$

and

$$
\pi_{2}^{*}(\beta \mid \alpha, \underline{X})=\frac{K^{r+a_{2}}}{\Gamma\left[r+a_{2}\right]} \beta^{r+a_{2}-1} e^{-\beta K}=\operatorname{Gamma}\left(r+a_{2}, K\right)
$$

where $K=\frac{1}{\alpha} \sum_{i=1}^{n}\left(e^{\alpha x_{i}}-1\right)+b_{2}$.

Now, we will generate random samples for $\alpha$ and $\beta$ from the conditional posterior densities. In case of $\beta$ we will generate values from $\operatorname{Gamma}\left(r+a_{2}, K\right)$, while the conditional posterior distributions of $\alpha$ in (21) is not known distribution, and the plot of it shows that it is similar to normal distribution, see Figure 1. Therefore, to generate random values of $\alpha$ from this

distribution, we use the Metropolis-Hastings method with normal proposal distribution. Algorithm 1 is proposed to compute the BEs for the parameters $\alpha, \beta$ 

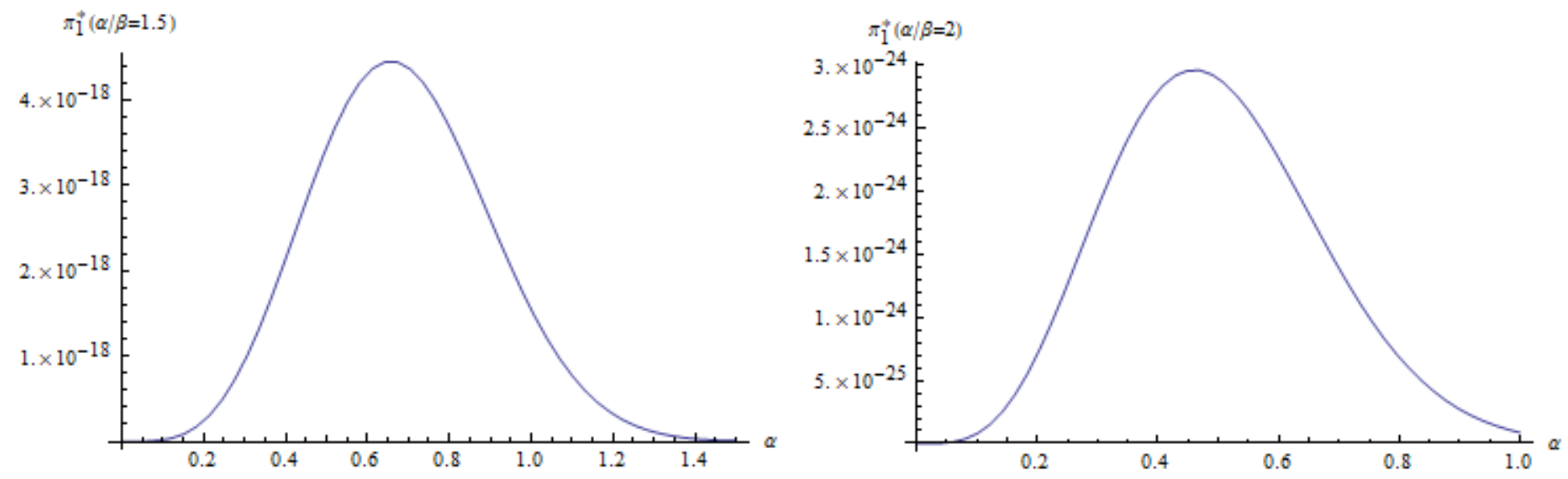

Figure 1: conditional posterior density of $\alpha$ when $\beta=1.5,2$ for a simulated sample with size $n=50$

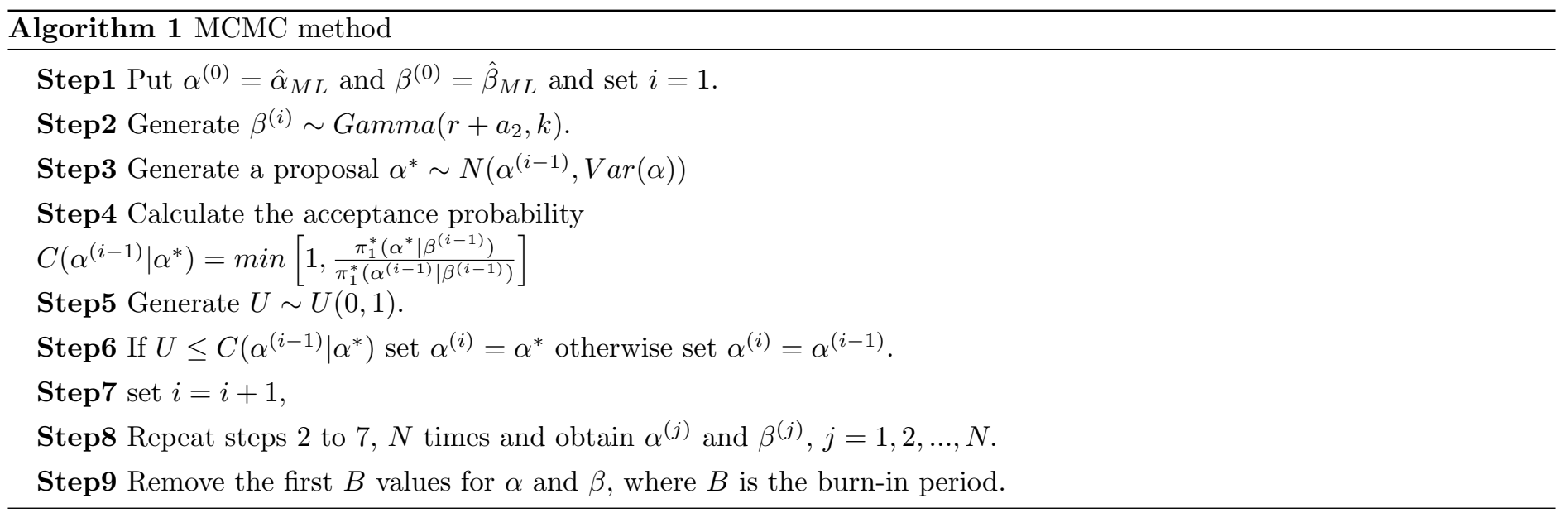


Based on the generated values for $\alpha$ and $\beta$ using Algorithm 1, we can obtain the BEs as follows; the estimates under $S E L$ are given by

$$
\begin{aligned}
& \tilde{\alpha}_{B S}=\frac{1}{N-B} \sum_{i=B+1}^{N} \alpha^{(i)} \\
& \tilde{\beta}_{B S}=\frac{1}{N-B} \sum_{i=B+1}^{N} \beta^{(i)}
\end{aligned}
$$

the BEs under $L I N E X$ are given by

$$
\begin{aligned}
& \tilde{\alpha}_{B L}=-\frac{1}{h} \log \left[\frac{1}{N-B} \sum_{i=B+1}^{N} e^{-h \alpha^{(i)}}\right] \\
& \tilde{\beta}_{B L}=-\frac{1}{h} \log \left[\frac{1}{N-B} \sum_{i=B+1}^{N} e^{-h \beta^{(i)}}\right]
\end{aligned}
$$

the BEs under GeneralizedEntropy are given by

$$
\begin{aligned}
& \tilde{\alpha}_{B E}=\left[\frac{1}{N-B} \sum_{i=B+1}^{N}\left(\alpha^{(i)}\right)^{-q}\right]^{-1 / q}, \\
& \tilde{\beta}_{B E}=\left[\frac{1}{N-B} \sum_{i=B+1}^{N}\left(\beta^{(i)}\right)^{-q}\right]^{-1 / q}
\end{aligned}
$$

Now, to compute the credible intervals for $\alpha$ and $\beta$ based on the generated values using Algorithm 1, sort the values for $\alpha$ ascending to be $\alpha_{(1)}, \alpha_{(2)}, \ldots, \alpha_{(N-B)}$, and also for $\beta$ to be $\beta_{(1)}, \beta_{(2)}, \ldots, \beta_{(N-B)}$, then $100(1-\gamma) \%$ credible intervals for $\alpha$ and $\beta$ are given as follows,

$$
\left(L_{\alpha}, U_{\alpha}\right)=\left(\alpha_{((N-B) \gamma / 2)}, \alpha_{((N-B)(1-\gamma / 2))}\right) \quad \text { and } \quad\left(L_{\beta}, U_{\beta}\right)=\left(\beta_{((N-B) \gamma / 2)}, \beta_{((N-B)(1-\gamma / 2))}\right)
$$

\section{One-Sample Bayesian Prediction Intervals}

In this section, we try to predict the unobserved values of lifetimes in the same samples using the Bayesian prediction intervals. Let $t_{1}, t_{2}, \ldots, t_{n}$ be the failure times for $\mathrm{n}$ units placed on the lifetime test follow $\operatorname{GOM}(\alpha, \beta)$. Based on multiply Type-I censoring the observations are $X_{(1)} \leq X_{(2)} \leq \ldots \leq X_{(s)} \leq \ldots \leq X_{(n)}$, where $X_{(s)}$ may equal the failure time $t_{s}$ or censoring time $T_{s}$. If $X_{(s)}=T_{s}$, then we need to predict the failure time for unit number $s$, which is $t_{s}$.

Let $K$ denote the number of unit failed before time $T_{s}$, where $K$ is a discrete random variable with Binomial probability given by,

$$
P(K=k)=\left(\begin{array}{c}
s-1 \\
k
\end{array}\right) p^{k} q^{s-1-k}, k=0,1, \ldots, s-1
$$

where $p=F\left(T_{s}\right)=1-\exp \left[-\frac{\beta}{\alpha}\left(e^{\alpha x}-1\right)\right]$ and $q=1-p$.

Now, the conditional density function of $X_{s}$, is given by

$$
\begin{aligned}
& f_{1}\left(x_{s} \mid \mathbf{x}_{k}\right)=\frac{1}{P(0 \leq K \leq s-1)} \sum_{k=0}^{s-1} f\left(x_{s} \mid \mathbf{x}_{k}, K=k\right) P(K=k) \\
& =\sum_{k=0}^{s-1} \frac{(n-k) ! \psi_{k}\left(T_{s}, \alpha, \beta\right)}{(s-k-1) !(n-s) !} \frac{\left[F\left(x_{s}\right)-F\left(T_{s}\right)\right]^{s-k-1}\left[1-F\left(x_{s}\right)\right]^{n-s} f\left(x_{s}\right)}{\left[1-F\left(T_{s}\right)\right]^{n-k}}, \quad T_{s}<x_{s}
\end{aligned}
$$


where $\psi_{k}\left(T_{s}, \alpha, \beta\right)=\frac{P(K=k)}{\sum_{k=0}^{s-1} P(K=k)}$.

Using (1)and (2) in (28), then the conditional density become,

$$
\begin{aligned}
f_{1}\left(x_{s} \mid \mathbf{x}_{k}\right) & =\sum_{k=0}^{s-1} \sum_{j=0}^{s-k-1} C(k) \psi_{k}\left(T_{s}, \alpha, \beta\right) \beta e^{\alpha x_{s}} \\
& \times \exp \left[-\frac{\beta}{\alpha}\left[\left(e^{\alpha x_{s}}-1\right)(n-k-j)+\left(e^{\alpha T_{s}}-1\right)(k+j-n)\right]\right],
\end{aligned}
$$

where $C(k)=\frac{(n-k) !(-1)^{s-k-1-j}}{j !(n-s) !(s-k-1-j) !}$

Assume that $\alpha$ and $\beta$ are unknown and having Gamma independent priors, as in (13). Using the posterior distribution $\pi^{*}(\alpha, \beta \mid \mathbf{x})$ in (14), then the predictive density function of $X_{s}$ is given by,

$$
f_{1}^{*}\left(x_{s} \mid \mathbf{x}_{k}\right)=\int_{0}^{\infty} \int_{0}^{\infty} f_{1}\left(x_{s} \mid \mathbf{x}_{k}\right) \cdot \pi^{*}\left(\alpha, \beta \mid \mathbf{x}_{k}\right) d \alpha d \beta, \quad T_{s}<x_{s}
$$

Since the integration in (30) is very complicated and can't be solved analytically, so the MCMC method is executed via algorithm [1] to get the approximates for these integrals, as follows;

$$
\begin{aligned}
f_{1}^{*}\left(x_{s} \mid \mathbf{x}_{k}\right) & =\frac{1}{N-B} \sum_{o=B+1}^{N} f_{1}\left(x_{s} \mid \mathbf{x}_{k}\right) \\
& =\frac{1}{N-B} \sum_{o=B+1}^{N} \sum_{k=0}^{s-1} \sum_{j=0}^{s-k-1} C(k) \psi_{k}\left(T_{s}, \alpha^{(o)}, \beta^{(o)}\right) \beta^{(o)} e^{\alpha^{(o)} x_{s}} \\
& \times \exp \left[-\frac{\beta^{(o)}}{\alpha^{(o)}}(n-k-j)\left(e^{\alpha^{(o)} x_{s}}-e^{\alpha^{(o)}} T_{s}\right)\right], \quad T_{s}<x_{s},
\end{aligned}
$$

Using (31), the predictive survival function of $X_{s}$ is given as follows,

$$
\begin{aligned}
\bar{F}_{1}^{*}\left(t \mid \mathbf{x}_{k}\right) & =\int_{t}^{\infty} f_{1}^{*}\left(x_{s} \mid \mathbf{x}_{k}\right) d x_{s} \\
& =\frac{1}{N-B} \sum_{o=B+1}^{N} \sum_{k=0}^{s-1} \sum_{j=0}^{s-k-1} \frac{C(k) \psi_{k}\left(T_{s}, \alpha^{(o)}, \beta^{(o)}\right)}{(n-k-j)} \times \exp \left[-\frac{\beta^{(o)}}{\alpha^{(o)}}(n-k-j)\left(e^{\alpha^{(o)}} t-e^{\alpha^{(o)}} T_{s}\right)\right] .
\end{aligned}
$$

Upon using (31), the Bayesian point predictor of $X_{s: n}$ under the SEL function is the mean of the predictive density, given by,

$$
\begin{aligned}
& \widehat{X}_{s: n}=\int_{T_{S}}^{\infty} x_{s} f_{1}^{*}\left(x_{s} \mid \mathbf{x}_{k}\right) d x_{s} \\
& =\frac{1}{N-B} \sum_{o=B+1}^{N} \sum_{k=0}^{s-1} \sum_{j=0}^{s-k-1} \frac{C(k) \psi_{k}\left(T_{s}, \alpha^{(o)}, \beta^{(o)}\right)}{(n-k-j)} \\
& \times\left(T_{s}+e^{\frac{\beta^{(o)}}{\alpha^{(o)}}\left(e^{\alpha^{(o)} T_{s}}-1\right)(n-k-j)} \int_{T_{s}}^{\infty} e^{-\frac{\beta^{(o)}}{\alpha^{(o)}}\left(e^{\alpha^{(o)} x_{s}}-1\right)(n-k-j)} d x_{s}\right)
\end{aligned}
$$

Also, the Bayesian prediction interval for $X_{s}, \quad k<s \leq n$ with $100(1-\gamma) \%$ is $\left[L_{X_{s}}, U_{X_{s}}\right]$, where $L_{X_{s}}$ and $U_{X_{s}}$ are obtained by solving the following two equations

$$
\bar{F}_{1}^{*}\left(L_{X_{s}} \mid \mathbf{x}_{k}\right)=1-\frac{\gamma}{2} \quad \text { and } \quad \bar{F}_{1}^{*}\left(U_{X_{s}} \mid \mathbf{x}_{k}\right)=\frac{\gamma}{2} .
$$




\section{Real Data Example}

In this section, we use the a set of real data that used by Chen [13], to get the estimates for the parameters $\alpha$ and $\beta$ and the corresponding asymptotic confidence bounds. The data are the numbers of tumor-days of 30 rats fed with unsaturated diet:

$60,63,63,63,66,66,66,68,70,70,77,77,84,91,91,94,98,101,105,108$

$109,112,112,115,126,143,153,161,164,178$.

Chen assumed that the population distribution of the numbers of tumor-free days is a Gompertz distribution with parameters $\alpha$ and $\beta$. Based on some goodness of fit tests, we can conclude that the given data follow $\operatorname{GOM}(0.024,0.0016)$, where these values are the MLEs in case of complete sample estimation. The following tests are used with the resulting P-value; the Kolmogorov-Smirnov (K-S)Test has p-value 0.18645, the Anderson-Darling test has P-value 0.18466 and the Pearson's chisquared test has $\mathrm{P}$-value 0.0806587 . We assume that the censoring times are $T_{i}=60+\Delta(i), \Delta=3, i=1,2,3, \ldots, n$. i.e. $T_{1}=63, T_{2}=66, \ldots, T_{30}=150$, then the observations under multiply Type-I censoring are:

$$
\begin{gathered}
60,63,63,63,66,66,66,68,70,70,77,77,84,91,91,94,98,101,105,108, \\
109,112,112,115,126,138,141,144,147,150 .
\end{gathered}
$$

with $r=\sum_{i=1}^{30} \delta_{i}=25$, then the condition in (9) is satisfied, because $2\left(\sum_{i=1}^{n} x_{i}\right)\left(\sum_{i=1}^{n} x_{i} \delta_{i}\right)$ equal 12391250 and $r \sum_{i=1}^{n}\left(x_{i}\right)^{2}$ equal 7491125, then the MLEs of the parameters $\alpha, \beta$ are exist and unique. The MLEs of $\alpha$ and $\beta$ are obtained numerically by solving the two equations (6) and (7) or graphically by drawing the function $J(\alpha)$ in (8) as obtained in figure (2) and the MlEs are given by $\hat{\alpha}_{M L}=0.02528$ and $\hat{\beta}_{M L}=0.001526$. Also the $95 \%$ asymptotic confidence bounds for $\alpha$ are $(0.01460,0.03597)$

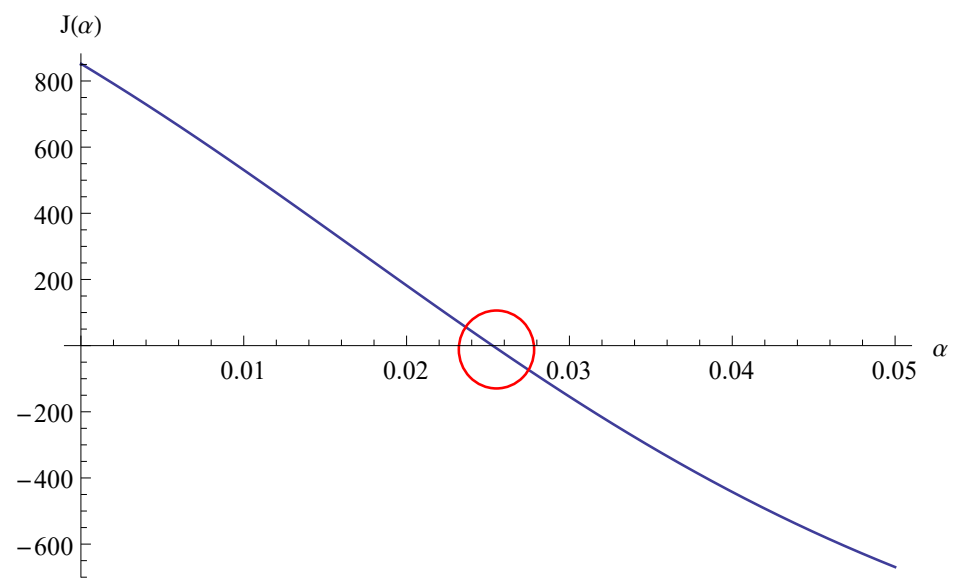

Figure 2: MLE of $\alpha$ when $J(\alpha)=0$

and the bounds for $\beta$ are $(-0.0000015 \simeq 0,0.00305)$. We also note that, the Gompertz distribution is a best fit for these real data when we apply the goodness of fit tests using the MLES. The BEs using MCMC for the parameters $\alpha$ and $\beta$ based on different loss functions. The number of iteration for the MCMC algorithm is $N=5000$ with the burn-in period $B=1000$. We choose the values of the hyper parameters to be $\left(a_{1}, b_{1}\right)=(0.0128,0.51)$ for $\alpha$ and $\left(a_{2}, b_{2}\right)=(0.000046,0.0305)$ for $\beta$ (by letting the mean of the Gamma prior distribution of $\alpha$ equal $\hat{\alpha}_{M L}$ and its variance is 0.05 i.e $a_{1}, b_{1}$ are the solution of the equations $a_{1} / b_{1}=0.02528$ and $a_{1} / b_{1}^{2}=0.05$, and the mean of the Gamma prior distribution of $\beta$ is equal to $\hat{\beta}_{M L}$ and its variance is 0.05 i.e $a_{2}, b_{2}$ are the solution of the equations $a_{2} / b_{2}=0.001526$ and $a_{2} / b_{2}^{2}=0.05$ ). Also we take $h=0.5$ and $q=-0.5$. Then the BEs are given in the following table. 


\begin{tabular}{|c|c|c|c|}
\hline Bayes method & SEL & LINEX & GE \\
\hline$\alpha$ & 0.02562 & 0.02561 & 0.02531 \\
\hline$\beta$ & 0.001605 & 0.001605 & 0.001501 \\
\hline
\end{tabular}

Also the $95 \%$ credible intervals for $\alpha$ and $\beta$ are given by $(0.01557,0.03692)$ and $(0.000447,0.00362)$, respectively. Based on the multiply Type-I censoring, the unobserved times (where $X_{j}>T_{j}$ ) are $X_{26}, X_{27}, X_{28}, X_{29}$ and $X_{30}$, so we will obtain the Bayesian point predictors and the One-sample Bayesian prediction bounds for them. Table 1 shows the Bayesian point predictor and the lower and upper limits of the unknown times with $95 \%$ coverage level.

\begin{tabular}{|l|c|c|}
\hline$X_{s}$ & Point predictor $\widehat{X}_{s: n}$ & $\left(L_{x_{s}}, U_{x_{s}}\right)$ \\
\hline$X_{26}$ & 153.75 & $(102.96,173.93)$ \\
$X_{27}$ & 157.51 & $(112.52,179.13)$ \\
$X_{28}$ & 161.87 & $(125.45,185.56)$ \\
$X_{29}$ & 167.48 & $(144.20,194.51)$ \\
$X_{30}$ & 176.82 & $(151.53,210.68)$ \\
\hline
\end{tabular}

Table 1: The 95\% One-sample Bayesian prediction intervals and the Bayesian point predictor for real data

\section{Simulation Study}

In this section, Monto Carlo simulation will be used to compare the performance of the proposed methods for estimating the parameters of Gompertz distribution. We will compare the MLEs and the BEs depending on three different loss functions, also we will study the performance of the asymptotic confidence intervals. The real values of the parameters $\alpha$ and $\beta$, sample size $n$ and the censored time points $T_{1}, T_{2}, \ldots, T_{n}$ are required by the simulation study.

The values of the parameters are chosen to be $0.5,1$ for $\alpha$ and $0.5,1,2$ for $\beta$ with sample size $n=10,30,50$. The censored time points will be chosen with equal difference $\Delta=\Delta_{i}=T_{i+1}-T_{i}$ equal 0.1 and 0.25 . The process of simulation will be executed 1000 times, each time we generate a multiply Type-I censored sample " $X_{1}, X_{2}, \ldots, X_{m}, \ldots, X_{n} \sim G O M(\alpha, \beta)$ " and the different estimates are calculated. Then the mean of 1000 values for the MLEs and BEs of the parameters $\alpha$ and $\beta$ are obtained, also the we obtain the mean of 1000 lower and upper confidence bounds for the asymptotic confidence intervals of the parameters with $95 \%$ confidence limits.

In the BEs, the MCMC method will be used according to Algorithm (1) that was mentioned in section 4. The number of iteration for this algorithm is $N=2000$ with the burn-in period $B=500$. Our estimates are obtained depending on the SEL loss function, the LINEX loss function with parameter $h=0.5$ and the GE with parameter $q=-0.5$. We assumed that the parameters $\alpha$ and $\beta$ have a gamma priors $\operatorname{Gamma}\left(a_{1}, b_{1}\right), \operatorname{Gamma}\left(a_{2}, b_{2}\right)$ respectively as in (13). The values of the hyper parameters with its variances are chosen to be.

\begin{tabular}{|c|c|c|c|c|c|}
\hline parameter & $\alpha=0.5$ & $\alpha=1$ & $\beta=0.5$ & $\beta=1$ & $\beta=2$ \\
hyperparameters $(a, b)$ & $(5,10)$ & $(10,10)$ & $(5,10)$ & $(10,10)$ & $(10,5)$ \\
$\operatorname{Var}($ parameter $)$ & 0.05 & 0.1 & 0.05 & 0.1 & 0.4 \\
\hline
\end{tabular}


We choose the hyper parameters by letting the mean of the Gamma prior distribution of $\alpha$ is real value of alpha i.e $\frac{a_{1}}{b_{1}}=$ real $\alpha$ and its variance is $\operatorname{Var}(\alpha)$ i.e $\frac{a_{1}}{b_{1}^{2}}=\operatorname{Var}(\alpha)$, and the same also for $\beta$. The results of this simulation is obtained in tables 2,3 and 4. In table 2, we present the bias of the estimate and the mean square error (MSE) for the MLEs and the BEs under three loss functions for $\alpha=0.5, \beta=0.5,1,2$. while table 3 , present these estimates for $\alpha=1, \beta=0.5,1,2$. The asymptotic confidence intervals and the credible intervals with $95 \%$ confidence degree for $\alpha=0.5,1$ and $\beta=0.5,1,2$ are included in table 4 .

\section{Concluding remarks}

In this paper, we study the estimates for the parameters of Gompertz distribution when the units under the lifetime test terminated at different times (Multiply Type-I censoring) using maximum likelihood method and Bayesian method under three types of loss functions (SEL, LINEX, GE). Also we have gotten the condition for the existence and uniqueness of the MLEs, also the asymptotic confidence intervals are constructed. Prediction intervals is conducted for the unobserved failures in the same sample. According to these results of simulation and the introduced examples, we can draw some conclusions:

- The estimators that obtained from Bayesian method are very close to the real values of parameters than the estimators of Maximum likelihood method.

- In most cases, the Bayes estimates using the LINEX loss function are better than those using the SEL or GE loss functions.

- The estimators that depend on samples with large sizes are better than those with small sizes.

- In most cases, we have obtained the best estimates for the largest difference of censoring time points $\Delta$ i.e results with $\Delta=0.25$ is better than that of $\Delta=0.1$.

- The asymptotic confidence intervals always include the MLE of $\alpha$ and $\beta$ and have positive values.

- When the sample size $n$ increased, the asymptotic confidence intervals and the credible intervals become more narrow.

- The credible intervals are more accurate and narrow than the asymptotic confidence intervals.

- The confidence intervals at $\Delta=0.25$ are more accurate than that constructed using $\Delta=0.1$.

- When $\alpha$ is known, then we can apply this work to exponential distribution using the transformation $Y=\frac{1}{\alpha}\left(e^{\alpha x}-1\right)$.

\section{Acknowledgments}

The authors are sincerely grateful to the referees and to the Editor-in-Chief for their many constructive comments and careful reading of the paper.

\section{References}

[1] N. Balakrishnan and R. Aggarwala. Progressive censoring: theory, methods, and applications. Springer Science \& Business Media, 2000. 
[2] N. Balakrishnan. Progressive censoring methodology: an appraisal. Test, 16(2):211-259, 2007.

[3] X. Jia, D. Wang, P. Jiang, and B. Guo. Inference on the reliability of weibull distribution with multiply type-i censored data. Reliability Engineering \& System Safety, 150:171-181, 2016.

[4] X. Jia and B. Guo. Exact inference for exponential distribution with multiply type-i censored data. Communications in Statistics-Simulation and Computation, (just-accepted):00-00, 2016.

[5] M. M. Mohie El-Din and A. R. Shafay. One-and two-sample bayesian prediction intervals based on progressively type-ii censored data. Statistical Papers, 54(2):287-307, 2013.

[6] A. R. Shafay and N. Balakrishnan. One-and two-sample bayesian prediction intervals based on type-i hybrid censored data. Communications in Statistics-Simulation and Computation, 41(1):65-88, 2012.

[7] M. M. Mohie El-Din, Y. Abdel-Aty, and A. R. Shafay. Two-sample bayesian prediction intervals of generalized order statistics based on multiply type ii censored data. Communications in Statistics-Theory and Methods, 41(3):381-392, 2012.

[8] M. M. Mohie El-Din, F. H. Riad, and M. A. El-Sayed. Statistical inference and prediction for the inverse weibull distribution based on record data. Journal of Statistics Applications \& Probability, 3(2):171, 2014.

[9] M. M. Mohie El-Din, M. S. Kotb, and H. A. Newer. Bayesian estimation and prediction for pareto distribution based on ranked set sampling. Journal of Statistics Applications \& Probability, 4(2):211, 2015.

[10] Y. Abdel-Aty. Bayesian prediction of future number of failures based on finite mixture of general class of distributions. Statistics, 46(1):111-122, 2012.

[11] B. Gompertz. On the nature of the function expressive of the law of human mortality, and on a new mode of determining the value of life contingencies. Philosophical transactions of the Royal Society of London, pages 513-583, 1825.

[12] J. H. Pollard and E. J. Valkovics. The gompertz distribution and its applications. Genus, pages 15-28, 1992.

[13] Z. Chen. Parameter estimation of the gompertz population. Biometrical Journal, 39(1):117-124, 1997.

[14] S. Chang and T. Tsai. Point and interval estimations for the gompertz distribution under progressive type-ii censoring. Metron, 61(3):403-418, 2003.

[15] J. W. Wu, W. L. Hung, and C. H. Tsai. Estimation of parameters of the gompertz distribution using the least squares method. Applied mathematics and computation, 158(1):133-147, 2004.

[16] M. M. Mohie El-Din, Y. Abdel-Aty, and M. H. Abu-Moussa. Statistical inference for the gompertz distribution based on type-ii progressively hybrid censored data. Communications in Statistics - Simulation and Computation, 46(8):6242$6260,2017$.

[17] M. E. Ghitany, F. Alqallaf, and N. Balakrishnan. On the likelihood estimation of the parameters of gompertz distribution based on complete and progressively type-ii censored samples. Journal of Statistical Computation and Simulation, 84(8):1803-1812, 2014.

[18] B. Efron and D. V. Hinkley. Assessing the accuracy of the maximum likelihood estimator: Observed versus expected fisher information. Biometrika, pages 457-482, 1978. 
[19] C. Robert and G. Casella. Monte Carlo Statistical Methods. Springer, New York, NY, USA, 2005.

[20] S. Upadhyay and A. Gupta. A bayes analysis of modified weibull distribution via markov chain monte carlo simulation. Journal of Statistical Computation and Simulation, 80(30):241-254, 2010.

[21] Z. Jaheen and M. Al Harbi. Bayesian estimation for the exponentiated weibull model via markov chain monte carlo simulation. Communications in Statistics: Simulation and Computation, 40(4):532-543, 2011. 


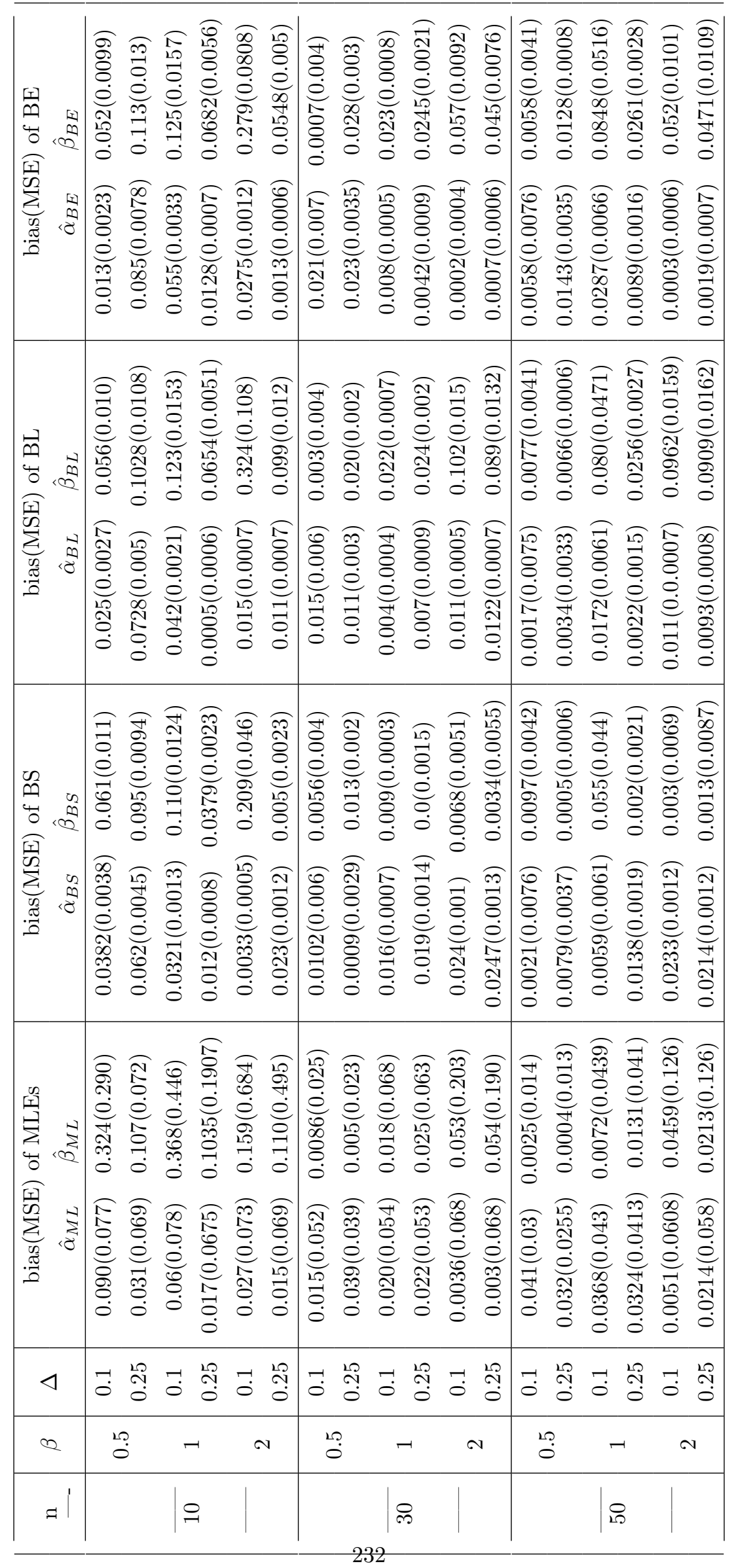

Table 2: MLEs, Bayes estimates using SEL (BS), LINEX (BL) and Generalized Entropy(BE) for $\alpha=0.5, \beta=0.5,1,2$ 


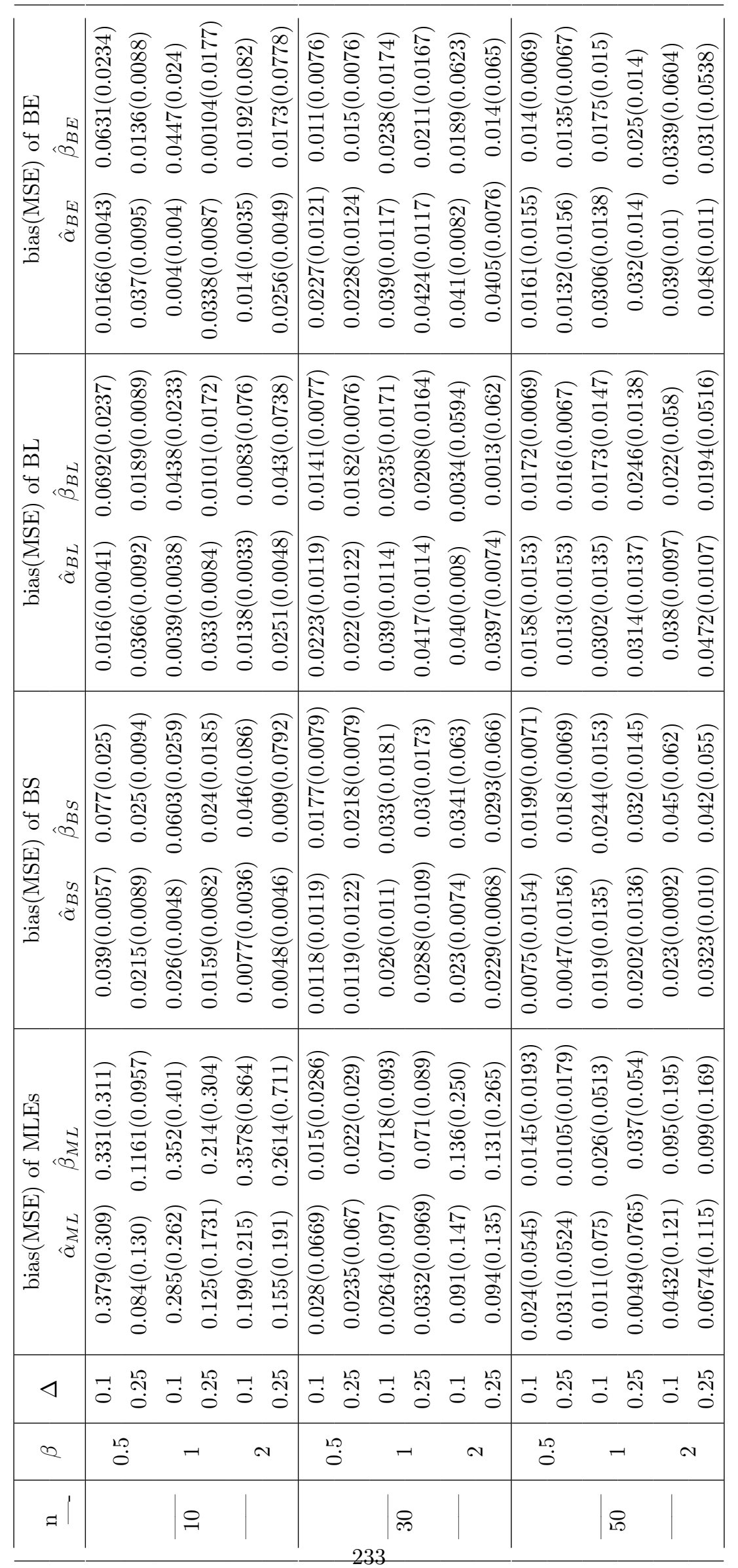

Table 3: MLEs, Bayes estimates using SEL (BS), LINEX (BL) and Generalized Entropy(BE) for $\alpha=1, \beta=0.5,1,2$ 


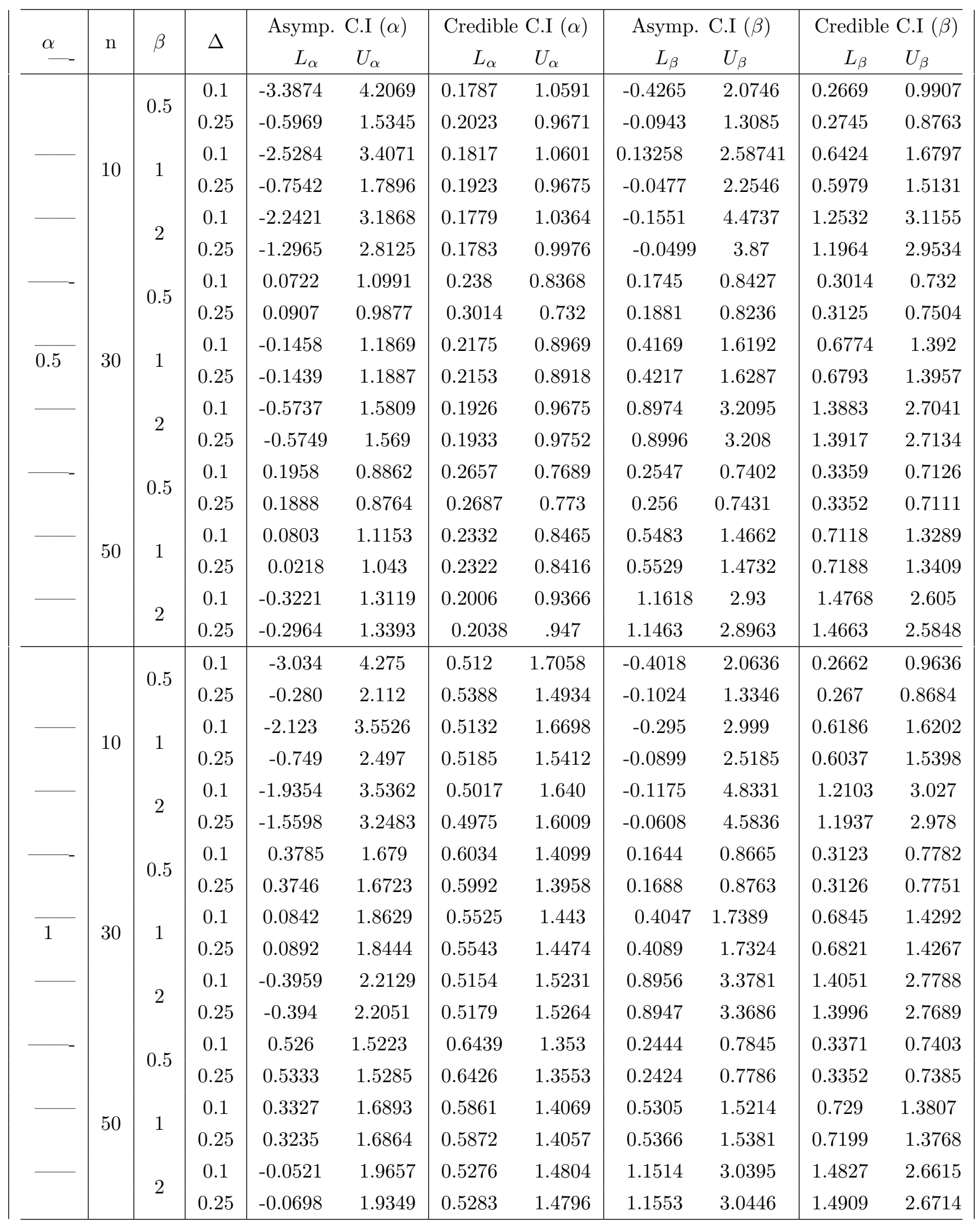

Table 4: asymptotic and credible confidence bounds for $\alpha=0.5,1, \beta=0.5,1,2$ 